Supplement Issue 1: International Tehran Breast Cancer Congress (TBCC9)

\title{
Comparison of Visual Assessment of Breast Density in BI-RADS 5th Edition with Automated Volumetric Measurement
}

DOI: $10.21859 / \mathrm{mci}-$ supp-114

\author{
Sima Ravanfar ${ }^{1, *}$ \\ ${ }^{1}$ Shiraz University of Medical Science \\ * Corresponding author: Sima Ravanfar, Shiraz University of Medical Science. \\ E-mail:sravanfarr@gmail.com
}

\section{Keywords:}

Breast

Density

Measurement

\begin{abstract}
Introduction: The purpose of this study was to compare visual assessment of mammographic breast density by radiologists using BI-RADS 5th edition in correlation with automated volumetric breast density measurements.
\end{abstract}

Materials and Methods: A total of 500 consecutive full-field digital mammographic examinations with standard views were retrospectively assessed by two radiologists for mammographic breast density according to BI-RADS 5th edition. Fully automated measurement of the volume of fibroglandular tissue, total breast and percentage breast density was performed with an available software program. Interobserver and intraobserver agreement was assessed with kappa statistics. The distributions of breast density categories for 5th edition of BI-RADS was correlated with volumetric data.

Results: Interobserver agreement on breast density category was moderate to substantial with use of the 5th edition. All volumetric breast density data were significantly different among density categories and had significant correlation with visual assessment for 5 th edition of BI-RADS $(\mathrm{p}<0.01)$.

Conclusions: Automated volumetric density assessment had good correlation with visual assessment for 5 th edition of BI-RADS. 